\title{
Investigation of Bias Current and Modulation Frequency Dependences of Detectivity of YBCO TES and the Effects of Coating of $\mathrm{Cu}-\mathrm{C}$ Composite Absorber Layer
}

\author{
Ali Moftakharzadeh, Alireza Kokabi, Ali Bozbey, Tayebeh Ghodselahi, Mohammad Ali Vesaghi,
} Sina Khorasani, Senior Member, IEEE, Marko Banzet, Juergen Schubert, and Mehdi Fardmanesh, Senior Member, IEEE

\begin{abstract}
Bolometric response and noise characteristics of YBCO superconductor transition edge IR detectors with relatively sharp transition and its resulting detectivity are investigated both theoretically and experimentally. The magnitude of response of a fabricated device was obtained for different bias currents and modulation frequencies. Using the measured and calculated bolometric response and noise characteristics, we found and analyzed the device detectivity versus frequency for different bias currents. The detectivity versus chopping frequency of the device did not decrease following the response strongly, due to the decrease of the noise at higher frequencies up to $1 \mathrm{kHz}$, resulting in maximum detectivity around the modulation frequency of $100 \mathrm{~Hz}$. We also improved the responsivity of the device through the increase of the surface absorption by using a novel infrared absorber, which is made of a copper-carbon composite, coated in a low-temperature process. Within the modulation frequency range studied in this paper, comparison of device detectivity before and after coating is also presented.
\end{abstract}

Index Terms-Detectivity, infrared absorber, transition edge sensor (TES).

\section{INTRODUCTION}

$\mathbf{S}$ INCE the early reports about the feasibility of using high$T_{c}$ superconductors as a bolometer [1], many works have

Manuscript received November 8, 2008; revised February 14, 2009. First published June 26, 2009; current version published July 29, 2009. This paper was recommended by Associate Editor M. Mueck. This work was supported in part by TUBITAK under Project 105E134.

A. Moftakharzadeh, A. Kokabi, and S. Khorasani are with the School of Electrical Engineering, Sharif University of Technology, Tehran 113659363, Iran (e-mail: ali_moftakhar@yahoo.com; alirezakokabi@gmail.com; khorasani@sharif.edu).

A. Bozbey is with the Department of Electrical and Electronics Engineering, TOBB University of Economics and Technology, Ankara 06560, Turkey (e-mail: bozbey@etu.edu.tr).

T. Ghodselahi is with the School of Physics, Institute for Studies in Theoretical Physics and Mathematics (IPM), Tehran 19395-5531, Iran (e-mail: ghodselahi@ipm.ir).

M. A. Vesaghi is with the Department of Physics, Sharif University of Technology, Tehran 11155-9161, Iran (e-mail: vesaghi@sharif.edu).

M. Banzet and J. Schubert are with the Jülich Research Center, Institute of Bio- and Nanosystems, 52425 Jülich, Germany (e-mail: m.banzet@fz-juelich. de; j.schubert@fz-juelich.de).

M. Fardmanesh was with the Electrical Engineering Department, Bilkent University, Ankara 06800, Turkey. He is now with the School of Electrical Engineering, Sharif University of Technology, Tehran 11365-9363, Iran (e-mail: fardmanesh@sharif.edu).

Digital Object Identifier 10.1109/TASC.2009.2020866 been focused on the applications of $\mathrm{YBCO}\left(\mathrm{YBa}_{2} \mathrm{Cu}_{3} \mathrm{O}_{7-x}\right)$ in different types of radiation detectors for the near-to-far-infrared wavelength regime [1]-[7]. Above $77 \mathrm{~K}$, probably the highest detectivity over the broadest band from the near to the far infrared can be achieved by high- $T_{c}$ superconducting transition edge bolometers [7]-[9], the physical operation principle of which is based upon the steep drop in their resistance $R$ at the transition temperature $T_{c}$ [10]-[12].

Due to the small noise level and high responsivity of this type of detector at its operating temperature, it can exhibit high detectivity values [8], [13]. The detectivity depends on the bias current and modulation frequency. These devices do not typically reach their potentially high detectivity values due to the lack of absorption of YBCO as well as the substrate, particularly in the micrometer range, leading to the use of an absorption layer. Some difficulties arise when using usual absorbers due to their destructive effects on the electrical properties of the superconducting YBCO material. For example, they can cause lowering the critical temperature $T_{c}$ or widening the transition curve that reduces the peak value in $d R / d T$ curves and hence reducing the IR response. YBCO thin film is very sensitive to high temperatures, destructive solvents, and stresses, hence limiting the suitable coating methods.

We investigated the effect of bias current and modulation frequency on device detectivity. This has been done theoretically by noise and response analysis and experimentally by measuring these two parameters at different values of bias currents and chopping frequencies. The lower frequency limit of the device is about $2 \mathrm{~Hz}$ due to extreme increase of the noise below it, and the higher frequency limit is about $10 \mathrm{kHz}$, which is the limit of our measurement system. We also proposed a novel absorber, which satisfies the aforementioned constraints and had very little destructive effect on our bolometer device. In the following, we also present the effect of this absorber coating on the responsivity and detectivity of the device.

\section{TheoreticAl Model}

The theoretical maximum detectivity of a bolometer is determined by both the responsivity and the voltage noise of the 
device through its noise equivalent power (NEP) as

$$
D^{*}=\frac{\sqrt{A}}{\mathrm{NEP}}=\frac{\sqrt{A(\Delta f)} r_{v}}{V_{n}} \mathrm{~cm} \cdot \mathrm{Hz}^{1 / 2} / \mathrm{W}
$$

where $A$ is the effective radiation absorption area of the detector, $r_{v}$ is the voltage responsivity, $V_{n}$ is the voltage noise, and $\Delta f$ is the frequency band used in the voltage noise measurement [14].

The voltage responsivity versus modulation frequency and temperature has previously been analyzed, which, according to the simple RC model, leads to [15]-[18]

$$
r_{v}=\frac{\eta L_{0}}{I_{b}\left(1-L_{0}\right) \sqrt{1+\left(2 \pi f \tau_{e}\right)^{2}}} \mathrm{~V} / \mathrm{W}
$$

where $L_{0}=\beta P_{b} / G_{t}$ is the electrothermal feedback gain coefficient, $P_{b}=I_{b}^{2} R_{b}$ is the bias power, $\beta=1 / R_{b} \times(d R / d T)$ is the temperature coefficient of resistance, $f$ is the modulation frequency, $\tau_{e}=\tau /\left(1-L_{0}\right)$ is the effective thermal time constant, $\tau=C_{t} / G_{t}$ is the thermal time constant, and $G_{t}$ and $C_{t}$ are the total thermal conductance and heat capacitance of the bolometer, respectively.

For high-frequency modeling, thermal diffusion length is defined as the characteristic penetration depth of the temperature variation into the substrate. Thermal diffusion length is determined from

$$
L=\sqrt{\frac{k_{s}}{\pi f c_{s}}}
$$

where $c_{s}$ and $k_{s}$ are the specific heat per unit volume and thermal conductivity of the substrate material, respectively [19]. Above a certain frequency, called knee frequency, thermal diffusion length becomes comparable to or smaller than the substrate thickness, and therefore, $G_{t}$ and $C_{t}$ are determined by modulation frequency as [19]

$$
\begin{aligned}
G_{t} & =A \sqrt{\pi f k_{s} c_{s}} \\
C_{t} & =A \sqrt{\frac{k_{s} c_{s}}{\pi f} .}
\end{aligned}
$$

The aforesaid consideration of $G_{t}$ and $C_{t}$ in the analysis results in a good approximation for the considered frequency range in this paper compared to that of the comprehensive 1-D model introduced in [19]. As seen from (3), the voltage responsivity of devices decreases due to the increase in chopping frequency [17].

Various intrinsic noise sources in addition to Johnson noise, phonon noise, and photon noise near the transition temperature are expected in a superconducting thin film. This class of noises is primarily due to magnetic flux penetration and fluctuations in the order parameter in the film [14], [20]-[23].

The total NEP for a high-temperature superconductor bolometer is obtained as follows [8], [15], [26]:

$$
\mathrm{NEP}^{2}=\frac{4 k_{B} T^{2} G_{t}}{\eta^{2}}+\frac{4 k_{B} T_{b} R_{b}}{r_{v}^{2}}+\frac{\alpha_{H} I_{b}^{2} R_{b}^{2}}{r_{v}^{2} N A d f}+\frac{8 \delta k_{B} A T_{B}^{5}}{\eta} .
$$

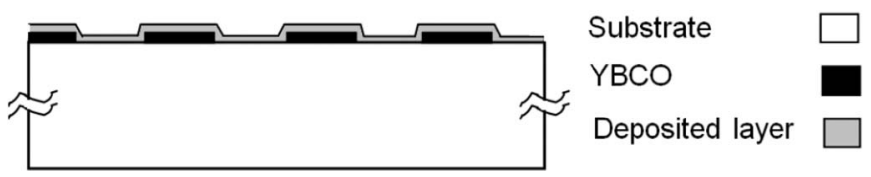

Fig. 1. Principal device structure with the $\mathrm{Cu}-\mathrm{C}$ absorber layer.

By considering (4) and (5), the dependence of responsivity versus chopping frequency can be obtained through (2) [19]. Substituting (6) using the result of (2) into (1), the spectrum of the detectivity of the device versus chopping is calculated. This approach is applied to compare the analytical and experimental results of detectivity in Section IV.

\section{EXPERIMENTAL SETUP}

The sample studied in this paper was made of 200-nm YBCO thin film on 1-mm-thick single-crystal $\mathrm{LaAlO}_{3}$ substrate. The YBCO film was deposited by pulsed laser deposition technique [28]. The device was patterned to meander line with track width and spacing of $100 \mu \mathrm{m}$. The samples were patterned using standard photolithography, and the etching process was carried on in about $0.75 \%$ dilute phosphoric acid. The active area of the patterns was $3.75 \mathrm{~mm}^{2}$. For the contact pads of the YBCO pattern, we deposited $\sim 50$-nm-thin gold layer after pre-etching YBCO using a dc planar magnetron etch-sputter unit. We used silver paste to make $\mathrm{Cu}$ wire contacts on the Au-coated pads. The sample was characterized before and after the deposition of the absorption layer on it. The final structure of the device is shown in Fig. 1, which will be further described and explained in the following sections.

The response of the sample was measured with a dc bias current $I_{\text {bias }}$ in four-probe configuration using an automated low-noise characterization setup. The temperature of the substrate was controlled with maximum $10-\mathrm{mK}$ deviation using a designed computer-based PID controller. The developed characterization system is also capable of sweeping temperature from $77 \mathrm{~K}$ to $95 \mathrm{~K}$ with given various rate values. By setting this rate to $2 \mathrm{~K} \cdot \mathrm{min}^{-1}$, we have minimized the effect of the temperature gradient and its associated error, which was caused by the temperature rise between the temperature sensor and the sample. The phase and magnitude response of the device were measured with a lock-in amplifier (SR830) using an ultralownoise preamplifier (SR560) at its input. The employed radiation source was a mechanically modulated (with a mechanical chopper) blackbody with a temperature of $640 \mathrm{~K}$. We have chosen this temperature due to the limitations of our setup.

The resistance of the device was measured simultaneously with the magnitude of the response in order to probe any deviation of magnitude of the response from the $d R / d T$ curve.

\section{EXPERIMENTAL RESULTS}

\section{A. Detectivity}

The theoretical explanation of detectivity was already discussed in the previous sections. The noise characteristics of this type of device versus temperature at different modulation 


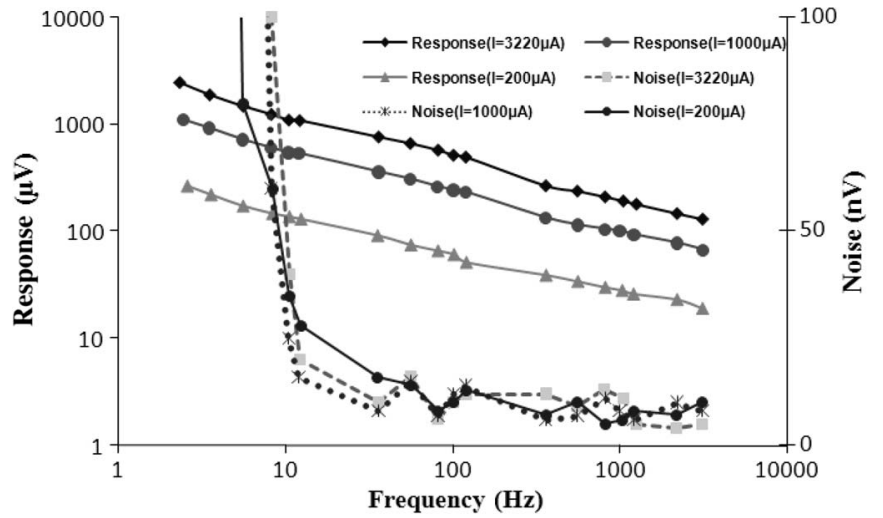

Fig. 2. (Left axis) Response and (right axis) noise for three different bias currents versus modulation frequency.

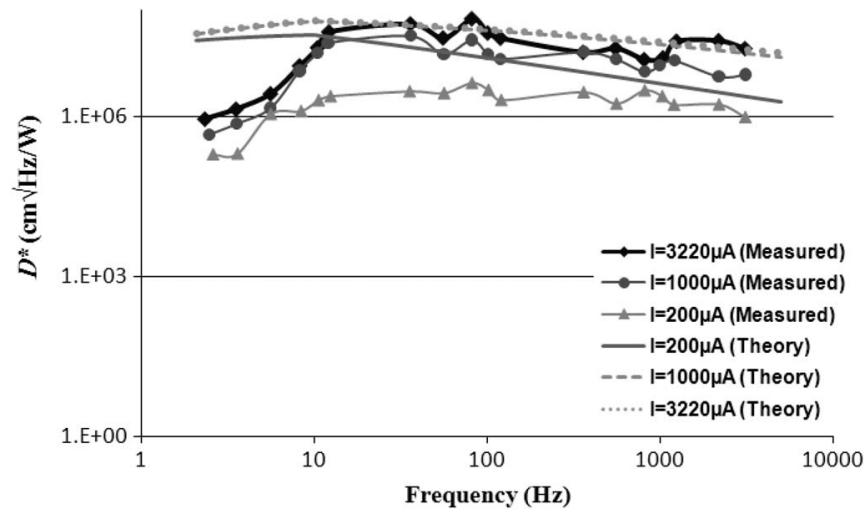

Fig. 3. Detectivity versus frequency for three different bias currents.

frequencies were also reported previously [12]. Here, we present the results of the measurements for the IR bolometric response and the noise versus modulation frequency at different bias currents of the device studied in this paper, as shown in Fig. 2. The related comprehensive results and analysis are reported in [11]. Fig. 2 shows that there is a low dependence of noise signal on the bias current. It also shows that noise signal below $10 \mathrm{~Hz}$ is considerably high. Therefore, the detectivity is perceptually low, where we have acquired maximum response at this range of frequency. Above $10 \mathrm{~Hz}$, the obtained detectivity versus chopping frequency of the device did not decrease considerably.

The observed result is shown in Fig. 2, in which the diminishing behaviors of response and noise versus frequency almost compensate each other above $10 \mathrm{~Hz}$, and we observe a nearly constant detectivity above $10 \mathrm{~Hz}$ over the investigated frequency range.

The results of the calculated and measured detectivities versus modulation frequency are shown in Fig. 3. This figure shows that the measured detectivity curves have many similarities to the theoretically predicted ones, i.e., in both of them, increasing bias current at its low values improves detectivity, while it has a very slight effect at higher values. This is due to the fact that, at low bias current where the device response is very small, the thermal NEP has the major effect on the total NEP of the device. However, by increasing the bias current, the effect of thermal NEP would decrease until the point that the total NEP would be limited by phonon NEP, which is independent of bias current.

Both calculated and measured detectivities exhibit little variation in the investigated frequency range. Below $10 \mathrm{~Hz}$, detectivity falls under its average values of higher frequencies in spite of large response over the low-frequency range. In the theoretical model, the slope of detectivity variation in this frequency range is $20 \mathrm{~dB} / \mathrm{dec}$. However, the measured slope is considerably higher, and the dependence of NEP to frequency does not follow the $1 / f$ type, which is under further investigation.

Fig. 3 shows a lower value for the measured detectivity at $200 \mu \mathrm{A}$ bias current with respect to the theoretical calculation, particularly at low frequencies. This might be associated to the granularity of the film that manifests itself at very low bias currents, increasing the contribution of $1 / f$-type noise component [13], [14].

\section{B. Coating}

In order to increase the NEP merit of devices, we worked on increasing the absorption coefficient. A YBCO material has a weak absorption coefficient in the IR range [14], and surface coating is essential for increasing the infrared response, particularly in the micrometer wavelength range.

We deposited a novel absorber layer over the area of the patterned YBCO thin film and unpatterned parts of the substrate, as seen in Fig. 1. This configuration increases not only the absorption coefficient of devices but also the effective absorbing area.

The absorber used in this paper is a 100-nm copper-carbon composite that is coated in a low-temperature planar magnetron sputtering process. The copper-carbon film was prepared by a capacitive coupled RF plasma-enhanced chemical vapor deposition system with $13.56-\mathrm{MHz}$ power supply. The reactor consists of two electrodes with different area sizes. The smaller electrode was a copper metallic disk with 75 -mm diameter as the powered electrode. The other electrode with $13-\mathrm{cm}$ diameter was grounded via the body of the stainless steel chamber. Deposition was performed on this electrode at room temperature. The chamber was evacuated to a base pressure of about $10^{-5}$ torr prior to the deposition process and was then raised to ambient pressure with controlled flow of pure acetylene gas. Deposition of the film was performed in two steps. First, a carbon layer was grown at $40-\mathrm{W}$ power, 50-mtorr process pressure, and 30-min deposition time, resulting in about 100-nm thin layer. Then, the copper-carbon layer was grown on it. For deposition of copper-carbon layer, RF power was $200 \mathrm{~W}$, and process pressure was set at 25 mtorr controlled by the gas flow. For a deposition time of $45 \mathrm{~min}$, a thickness of $100 \mathrm{~nm}$ was obtained for the $\mathrm{Cu}-\mathrm{C}$ composite film, resulting in a deposition rate of about $2.2 \mathrm{~nm} / \mathrm{min}$. The copper and carbon atomic contents of the film were obtained from RBS analysis and found to be 55\% and $45 \%$ for $\mathrm{Cu}$ and $\mathrm{C}$, respectively. By the control of the flow of acetylene and sputtering rate of $\mathrm{Cu}$ target, it is possible to produce carbon films with different $\mathrm{Cu}$ compositions.

Coatings may affect the device response in three different aspects. First, it can favorably increase the absorption coefficient and, therefore, temperature variation caused by same incident 


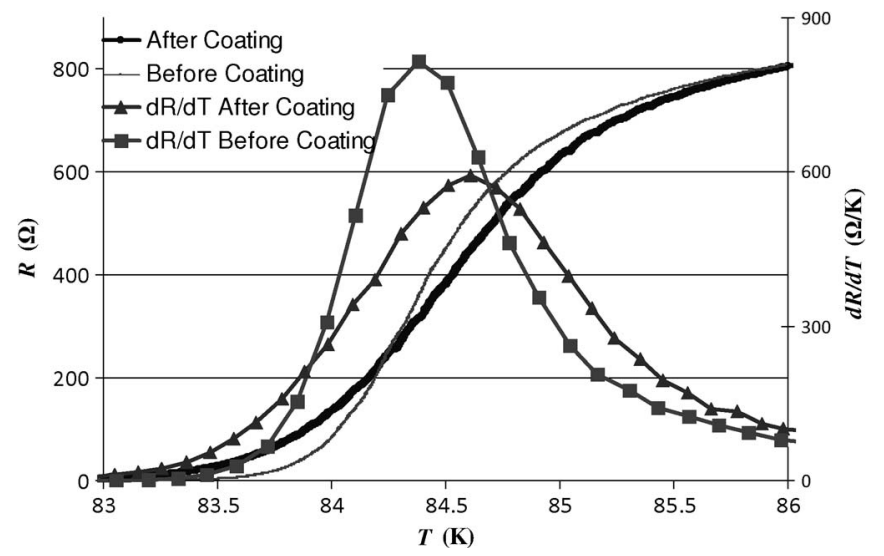

Fig. 4. (Left axis) Transition and (right axis) $d R / d T$ versus temperature at $100-\mu \mathrm{A}$ bias current.

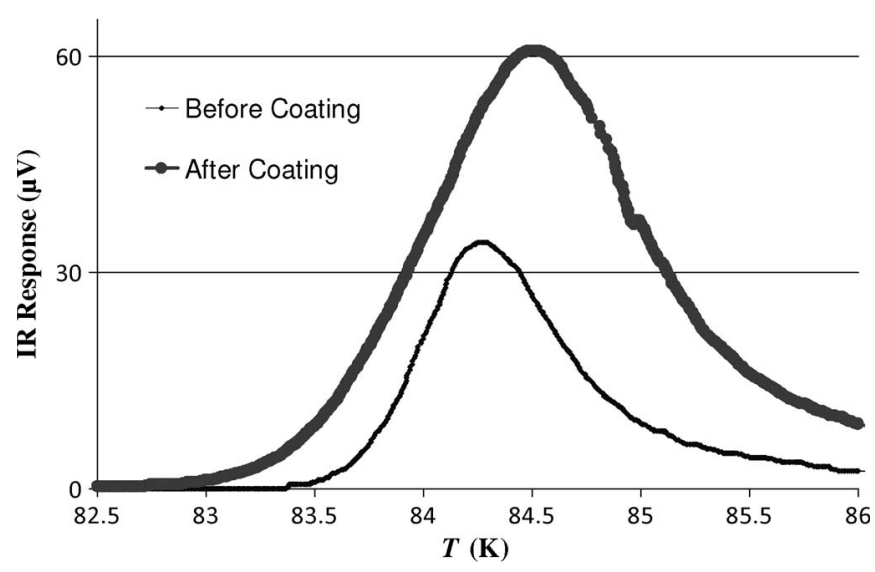

Fig. 5. IR response versus temperature at $100-\mu \mathrm{A}$ bias current, $48-\mathrm{Hz}$ chopping frequency, and wavelength of $4.5 \mu \mathrm{m}(640 \mathrm{~K})$.

infrared power, resulting in higher response and higher $D^{*}$. Second, it might smoothen the transition curve due to degradation of YBCO and so decrease $d R / d T$. Third, deposition of absorbing material may diminish the film quality and thus increase the noise level of the device. The last two effects are not desirable as they decrease device detectivity according to (1).

In Fig. 4, we observe that, after coating, the transition has very slightly widened, and therefore, the maximum value for $d R / d T$ has dropped. This indicates that we have some degradation of the YBCO film after coating. In Fig. 5, we present the measured value of IR response before and after coating. According to (2), since both measurements are done at the same bias current and chopping frequency, the following relation, which is based on $d R / d T$ and surface absorption, can be used to derive the responsivity variations:

$$
\frac{r_{v, \text { after coating }}}{r_{v \text {, before coating }}}=\frac{\eta_{\text {after coating }}}{\eta_{\text {before coating }}} \times \frac{(d R / d T)_{\text {after coating }}}{(d R / d T)_{\text {before coating }}} .
$$

By comparing the results of Figs. 4 and 5 and according to (7), it is found that the total surface absorption has increased by a factor of 2.4. Knowing the original device absorption to be about $7 \%$, the effective absorption of the copper-carbon thin layer could be approximated to be about $10 \%$ at the radiation wavelength peak of $4.5 \mu \mathrm{m}$. This approximation for the value of

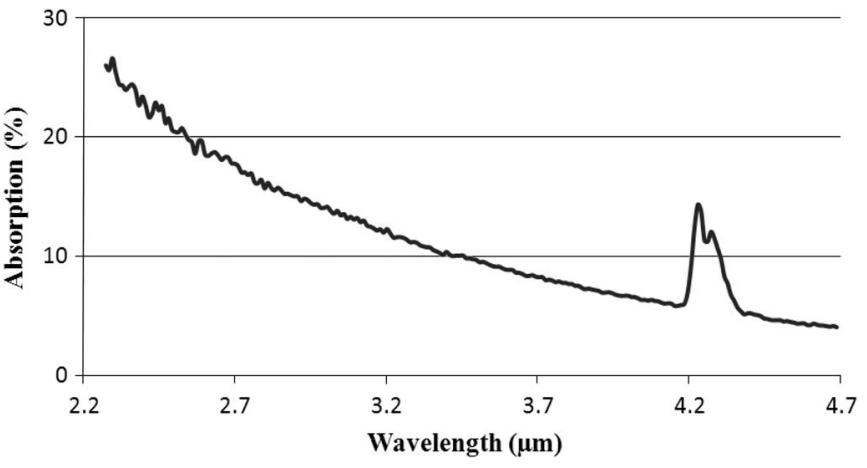

Fig. 6. IR spectroscopy of copper-carbon composite.

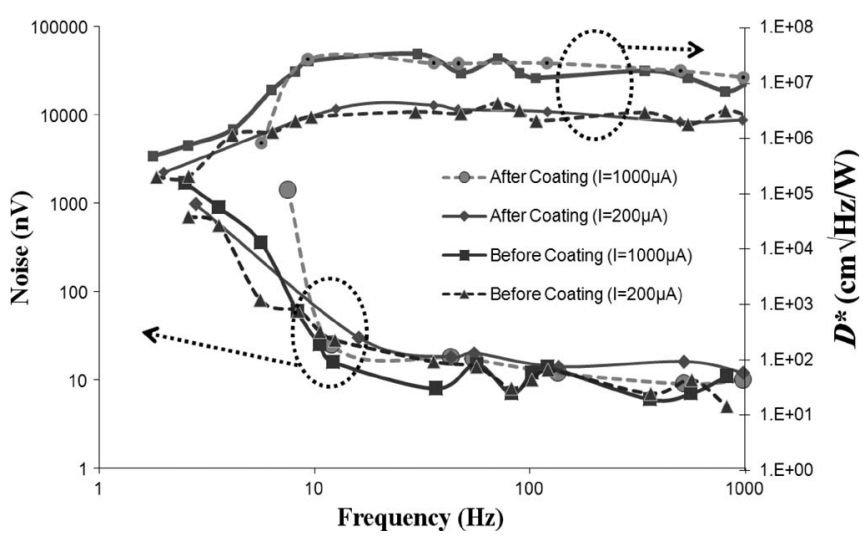

Fig. 7. (Left axis) Noise and (right axis) detectivity comparison of the device before and after coating.

absorption is the average of the overall absorption over the spectrum of the blackbody radiation. This approximately matches with our typical IR spectroscopy result for these composites, which is also shown in Fig. 6. The observed peak in absorption at $4.2 \mu \mathrm{m}$ in this figure might be partly associated to the special distribution and cluster size of the $\mathrm{Cu}$ species in the absorber layer, which is under further investigation.

The increase of absorption by the composite layer is associated to both the increase of the effective absorbing area and the total absorption of the layer in addition to the absorption of the YBCO film and the substrate. The effect of the increase of the absorbing area on response is with the consideration of the effect of lateral thermal diffusion length in the substrate and the film in the areas within the lines of the meander line of the YBCO film. Here, we suppose the overall absorption caused by the absorber layer to be effectively constant. This is found to be a good approximation for the considered frequency range since the thermal diffusion length for this range is larger than the spacing within the lines of the meander-line pattern of YBCO.

Due to slight degradation of the YBCO film, the noise of the device after coating increased (Fig. 7). In particular, in this case, which is our first try, this degradation has canceled out the increase of responsivity, and therefore, the detectivity is almost constant before and after coating, as shown in Fig. 7.

The change of copper doping in this type of composites strongly affects their absorption coefficient, which is under further investigation for finding the optimal doping level. 
As also noticed from the variations in the transition curve, in Fig. 5, there is a shift in maximum response with respect to the bias temperature. This phenomenon might be associated to the degradation of YBCO due to diffusion of carbon into the material affecting mainly in the lower part of the transition curve, which caused the similar effect on the $d R / d T$ curve shown in Fig. 4.

\section{CONCLUSION}

In this paper, we have measured and calculated the bolometric response and the noise signal over the interest range of modulation frequency and obtained its associated detectivity. Our investigation showed that the detectivity was nearly constant in the middle range of frequency and considerably dropped at lower frequencies due to higher noise level in this range. This is associated to the considerable increase of the $1 / f$-type noise in our device. The measured values for detectivity well matched the expected theoretical values. We also used a new $\mathrm{Cu}-\mathrm{C}$ composite absorber on the bolometers to increase the $D^{*}$ of devices in the measured micrometer radiation range. The coating of the absorber had no major destructive effect on the electrical characteristics of the device, degrading $d R / d T$ slightly. The used thickness of the absorbing layer was as low as about $100 \mathrm{~nm}$, and no phase lag and thermal damp were observed for the investigated modulation frequency range. Because of the high percentage of copper in the trial first-run deposited composite used in this paper, the absorption coefficient was improved within a factor of two. A detectivity $D^{*}$ in the range of $10^{8} \mathrm{~cm} \cdot \mathrm{Hz}^{0.5} / \mathrm{W}$ at about $100-\mathrm{Hz}$ modulation frequency was obtained for a blackbody radiation signal with a peak value at about $4.5 \mu \mathrm{m}$. The effect of copper doping on detectivity as well as the degradation of $d R / d T$ is under further investigation.

\section{REFERENCES}

[1] P. L. Richards, J. Clarke, R. Leoni, P. Lerch, S. Verghese, M. R. Beasley, T. H. Geballe, R. H. Hammond, P. Rosenthal, and S. R. Spielman, "Feasibility of the high $T_{c}$ superconducting bolometer," Appl. Phys. Lett., vol. 54, no. 3, pp. 283-285, Jan. 1989.

[2] F. Rahman, "Superconducting detectors in astronomy," Contemp. Phys., vol. 47, no. 4, pp. 181-194, Aug. 2006.

[3] J. Delerue, A. Gaugue, P. Teste, E. Caristan, G. Klisnick, A. Redon, and A. Kreisler, "YBCO mid-infrared bolometer arrays," IEEE Trans. Appl. Supercond., vol. 13, no. 2, pp. 176-179, Jun. 2003.

[4] J. C. Brasunas and B. Lakew, "High $T_{c}$ superconductor bolometer with record performance," Appl. Phys. Lett., vol. 64, no. 6, pp. 777-778, Feb. 1994

[5] Q. Li, D. B. Fenner, W. D. Hamblen, and W. G. Hamblen, "Epitaxial $\mathrm{YBa}_{2} \mathrm{Cu}_{3} \mathrm{O}_{7-y}$ bolometers on micromachined windows in silicon wafers," Appl. Phys. Lett., vol. 62, no. 19, pp. 2428-2430, May 1993.

[6] N. Bluzer, "Analysis of quantum superconducting kinetic inductance photodetectors," J. Appl. Phys., vol. 78, no. 12, pp. 7340-7351, Dec. 1995.

[7] S. J. Berkowitz, A. S. Hirahara, K. Char, and E. N. Grossman, "Low noise high-temperature superconducting bolometers for infrared imaging," Appl. Phys. Lett., vol. 69, no. 14, pp. 2125-2127, Sep. 1996.

[8] M. J. M. E. de Nivelle, M. P. Bruijn, R. de Vries, J. J. Wijnbergen, and P. A. J. de Korte, "Low noise high- $T_{c}$ superconducting bolometers on silicon nitride membranes for far-infrared detection," J. Appl. Phys., vol. 82, no. 10, pp. 4719-4726, Nov. 1997.

[9] L. Mechin, J. C. Villegier, and D. Bloyet, "Suspended epitaxial YBaCuO microbolometers fabricated by silicon micromachining: Modeling and measurements," J. Appl. Phys., vol. 81, no. 10, pp. 7039-7047, May 1997.

[10] H. Kraus, "Superconductive bolometers and calorimeters," Supercond. Sci. Technol., vol. 9, no. 10, pp. 827-842, Jun. 1996.
[11] M. Fardmanesh, K. Scoles, and A. Rothwarf, "DC characteristics of patterned $\mathrm{YBa}_{2} \mathrm{Cu}_{3} \mathrm{O}_{7-x}$ superconducting thin-film bolometers: Artifacts related to Joule heating, ambient pressure, and microstructure," IEEE Trans. Appl. Supercond., vol. 8, no. 2, pp. 69-78, Jun. 1998.

[12] Q. Hu and P. L. Richards, "Design analysis of a high $T_{c}$ superconducting microbolometer," Appl. Phys. Lett., vol. 55, no. 23, pp. 2444-2446, Dec. 1989.

[13] A. Kokabi, A. Moftakharzadeh, and M. Fardmanesh, "Investigation of the bias current and frequency dependence of the detectivity of high- $T_{c}$ superconductor transition-edge IR detectors," presented at the Int. Conf. Electrical Engineering, Tehran, Iran, 2007.

[14] M. Fardmanesh, A. Rothwarf, and K. J. Scoles, "Noise characteristics and detectivity of $\mathrm{YBa}_{2} \mathrm{Cu}_{3} \mathrm{O}_{7}$ superconducting bolometers: Bias current, frequency, and temperature dependence," J. Appl. Phys., vol. 79, no. 4, pp. 2006-2011, Feb. 1996.

[15] I. A. Khrebtov, Noise of High Temperature Superconducting Bolometers, vol. 151. Berlin, Germany: Springer-Verlag, 2005, pp. 97-107.

[16] M. Nahum, S. Verghese, P. L. Richards, and K. Char, "Thermal boundary resistance for $\mathrm{YBa}_{2} \mathrm{Cu}_{3} \mathrm{O}_{7-\delta}$ films," Appl. Phys. Lett., vol. 59, no. 16 , pp. 2034-2036, Oct. 1991.

[17] A. Bozbey, M. Fardmanesh, I. N. Askerzade, M. Banzet, and J. Schubert, "Effects of the superconductivity transition on the response of YBCO edge transition bolometers," Supercond. Sci. Technol., vol. 16, no. 12, pp. 1554-1558, Nov. 2003.

[18] K. Repsas, A. Laurinavicius, A. R. Vaskevicius, and F. Anisimovas, "Response of superconducting $\mathrm{Y}-\mathrm{Ba}-\mathrm{Cu}-\mathrm{O}$ films to millimeter wave radiation," Appl. Phys. Lett., vol. 79, no. 27, pp. 4544-4546, Dec. 2001.

[19] M. Fardmanesh, "Analytic thermal modeling for dc-to-midrange modulation frequency response of thin-film high- $T_{c}$ superconductive edgetransition bolometers," Appl. Opt., vol. 40, pp. 1080-1088, Mar. 2001.

[20] S. F. Karmanenko, A. A. Semenov, V. N. Leonov, A. V. Bobyl, A. I. Dedoborets, A. V. Lunev, A. V. Nashchekin, and R. A. Suris, "Sources of flicker noise and the technology of superconducting microstripes based on yttrium barium cuprate films," Tech. Phys., vol. 45, no. 4, pp. 443-452, Apr. 2000.

[21] I. A. Khrebtov, "Noise properties of high temperature superconducting bolometers," Fluct. Noise Lett., vol. 2, no. 2, pp. R51-R70, Jun. 2002.

[22] M. Galeazzi, F. Zuo, C. Chen, and E. Ursino, "Intrinsic noise sources in superconductors near the transition temperature," Nucl. Instrum. Methods Phys. Res. A, Accel. Spectrom. Detect. Assoc. Equip., vol. 520, no. 1-3, pp. 344-347, Dec. 2003.

[23] E. R. Nowak, "Flux-flow voltage noise and normal-state resistance fluctuations in epitaxial (Dy, Y) $\mathrm{Ba}_{2} \mathrm{Cu}_{3} \mathrm{O}_{7}$ thin films," Phys. C, vol. 421, no. 1-4, pp. 15-34, Mar. 2005.

[24] F. N. Hooge, T. G. Kleinpenning, and L. K. Vandamme, "Experimental studies of 1/f noise," Rep. Prog. Phys., vol. 44, no. 5, pp. 479-532, May 1981.

[25] L. B. Kiss and P. Svedlindh, "Noise in high $T_{c}$ superconductors," IEEE Trans. Electron Devices, vol. 41, no. 11, pp. 2112-2122, Nov. 1994.

[26] I. A. Khrebtov and A. D. Tkachenko, "High-temperature superconductor bolometers for the IR region," J. Opt. Technol., vol. 66, no. 8, pp. 735741, Aug. 1999.

[27] M. Fardmanesh, K. J. Scoles, and A. Rothwarf, "Control of the responsivity and the detectivity of superconducting edge-transition $\mathrm{YBa}_{2} \mathrm{Cu}_{3} \mathrm{O}_{7-X}$ bolometers through substrate properties," Appl. Opt., vol. 38, no. 22, pp. 4735-4742, Aug. 1999.

[28] J. Schubert, M. Siegert, M. Fardmanesh, W. Zander, M. Prömpers, C. Buchal, J. Lisoni, and C. H. Lei, "Superconducting and electro-optical thin films prepared by pulsed laser deposition technique," Appl. Surf. Sci., vol. 168 , no. $1-4$, pp. $208-214$, Dec. 2000.

Ali Moftakharzadeh was born in Yazd, Iran, on October, 1981. He received the B.Sc. degree in electrical engineering from K. N. Toosi University of Technology, Tehran, Iran, and the M.Sc. degree in electrical engineering from Sharif University of Technology, Tehran, in 2004, where he is currently working toward the Ph.D. degree in electrical engineering.

$\mathrm{He}$ is a member of the Superconductive Electronics Research Laboratory, School of Electrical Engineering, Sharif University of Technology. His research interests include cryogenic system design, Josephson-junction-based devices, quantum computation, quantum optics, and superconductor bolometers. 
Alireza Kokabi was born in Iran in 1982. He received the B.S. and M.S. degrees in electrical engineering from Sharif University of Technology, Tehran, Iran, in 2006, where he is currently working toward the Ph.D. degree in electrical engineering.

$\mathrm{He}$ is a member of the Superconductive Electronics Research Laboratory (SERL), School of Electrical Engineering, Sharif University of Technology. His research interests include superconductivity, cryogenic system design, superconductor bolometers, superconducting quantum interference devices (SQUIDs), SQUID-based systems, and solid-state and photonic devices.

Ali Bozbey was born in Isparta, Turkey, on September 27, 1979. He received the B.S., M.S., and Ph.D. degrees in electrical and electronics engineering from Bilkent University, Ankara, Turkey, in 2001, 2003, and 2006, respectively.

In 2002, he was a Guest Researcher with the Jülich Research Center, Jülich, Germany, and in 2007, he was a Postdoctoral Researcher with Nagoya University, Nagoya, Japan. Since 2008, he has been with the Department of Electrical and Electronics Engineering, TOBB University of Economics and Technology, Ankara, where he teaches in the areas of semiconductor and superconductor electronics. His research interests include design, modeling, and applications of transition edge sensors, superconducting quantum interference devices, and rapid single-flux-quantum-based circuits.

Tayebeh Ghodselahi was born in Iran in August 1973. She received the B.Sc. degree in physics from Sharif University of Technology, Tehran, Iran, in 1996, the M.Sc. degree in solid-state physics from Iran University of Science and Technology, Tehran, in 1999, and the Ph.D. degree in nanocondensed matter physics from Sharif University of Technology in 2008.

She was an Assistant for the establishment of a carbon laboratory and was a Researcher Student with the Institute for Studies in Theoretical Physics and Mathematics (IPM), Tehran, from 2003 until 2008. She is currently a Postdoctoral Researcher with the School of Physics, IPM. Her research interests include diamondlike carbon (DLC) films; copper and nickel nanoparticles in DLC films; optical, electrical, and electronic properties of these films; surface plasmon resonance of metallic nanoparticles; metal-nonmetal transition; fabrication of optical, biological and gas sensors using films containing metal nanoparticles.

Mohammad Ali Vesaghi was born in Tehran, Iran, in 1947. He received the B.Sc. degree from Sharif University of Technology, Tehran, in 1971, and the M.S. and Ph.D. degrees in physics from the University of Chicago, Chicago, IL, in 1976 and 1981, respectively.

From 1981 to 1988, he was a Manager, a Member of the Board of Directors, and a Technical Advisor of some industrial public companies. Since 1988, he has been with Sharif University of Technology, where he is currently an Associate Professor in the Department of Physics. He has been the Head of the department for three years and was the Vice Chairman of the department twice. His research interests include study of physical properties of nanoparticles and nanotubes, semiconductor physics, Neutron Transmutation Doping applications, carbon thin films such as DLC and device barriers, solar cells, and applied superconductivity. He has more than 60 publications in international journals and various international and national conference proceedings.

Sina Khorasani (M'04-SM'09) was born in Tehran, Iran, on November 25, 1975. He received the B.Sc. degree in electrical engineering from Abadan Institute of Technology, Abadan, Iran, in 1995, and the M.Sc. and Ph.D. degrees in electrical engineering from Sharif University of Technology, Tehran, in 1996 and 2001, respectively.

After spending a two-year term as a Postdoctoral Fellow with the School of Electrical and Computer Engineering, Georgia Institute of Technology, Atlanta, he returned to Sharif University of Technology, where he is currently an Associate Professor of electrical engineering in the School of Electrical Engineering. He has authored or coauthored more than 140 contributions in journals and conference proceedings in various fields of optics and photonics, plasma physics, and solid-state electronics.
Marko Banzet was born in Dinslaken, Germany, in 1973. He received the degree in microelectronics and fabrication.

In 1989, he became a Physics Laboratory Assistant with the Jülich Research Center, Jülich, Germany, where since 1992, he has been a Technician in the fields of superconductivity, thin-film deposition, ion beam etching, lithographic structuring, and cleanroom technology. He is currently with the Institute of Bio- and Nanosystems, Jülich Research Center, as an Information Technology Engineer, participating in vocational training.

Juergen Schubert was born in Köln, Germany, in 1958. He received the Diploma degree in physics and the Ph.D. degree from the University Köln, Köln, Germany, in 1985 and 1989, respectively.

In 1984, he joined the Jülich Research Center, Jülich, Germany. From 1985 to 1989, he developed a high-pressure sputter technique for the growth of hightemperature superconductor thin films. Since then, he has been the Leader of the Laserlab of the IBN 1-IT, Jülich Research Center, Institute of Bio- and Nanosystems, and he is responsible for the growth of epitaxial oxide thin films (superconducting, ferroelectric, optical transparent, conducting, etc.) using the pulsed laser deposition method. In 2002, he spent one year at The Pennsylvania State University, University Park, as a Guest Scientist in the Oxide Molecular Beam Epitaxy Group of D. Schlom.

Mehdi Fardmanesh (SM'02) was born in Tehran, Iran, in 1961. He received the B.S. degree in electrical engineering from Tehran Polytechnic University, Tehran, in 1987, and the M.S. and Ph.D. degrees in electrical engineering from Drexel University, Philadelphia, PA, in 1991 and 1993, respectively.

In 1989, he joined Drexel University, and until 1993, he conducted research in the development of thin- and thick-film high-temperature superconducting materials and devices, and the development of ultralow-noise cryogenic characterization systems, where he was awarded a research fellowship by the Ben Franklin Superconductivity Center in 1989. From 1994 to 1996, he was the Principal Manager for R\&D and the Director of a private sector research electrophysics laboratory, while also teaching in the Departments of Electrical Engineering and Physics of Sharif University of Technology in Tehran. In 1996, he joined the Department of Electrical and Electronics Engineering, Bilkent University, Ankara, Turkey, where he teaches courses in the area of solid-state electronics while also supervising the Superconductivity Research Laboratory. In 1998 and 1999, he was also invited to the Forschungszentrum Jülich, Institut für Schicht- und Ionentechnik, Jülich, Germany, where he pursued the development of low-noise high-Tc RF-superconducting-quantum-interference-device (SQUID)-based magnetic sensors. After establishment of an international collaboration between Bilkent University and the Jülich Research Center in the field of superconductivity by him, from 2000 to 2004, he was the Director of the joint project for the development of high-resolution high-Tc SQUID-based magnetic imaging system. Since 2000, he has also reestablished his activities at the School of Electrical Engineering, Sharif University of Technology, where he is currently the Head of the Department of Electronics. He set up the Superconductor Electronics Research Laboratory at Sharif University of Technology in 2003, and has been its Director since then. His research interests mainly include the design, fabrication, and modeling of high-temperature superconductor devices and circuits such as bolometers, microwave filters and resonators, Josephson junctions, and SQUID-based systems, in the areas of which he is holding several international patents. 